\title{
Energy Efficient Chain Based Routing Protocol for Orchard Wireless Sensor Network
}

\author{
Huarui Wu $\mathrm{W}^{1,2,3} \cdot$ Huaji Zhu ${ }^{1,2,3} \cdot$ Lihong Zhang ${ }^{1,2,3} \cdot$ Yuling Song ${ }^{4}$
}

Received: 23 October 2018 / Revised: 9 April 2019 / Accepted: 11 May 2019 / Published online: 5 June 2019

(c) The Author(s) 2019

\begin{abstract}
Wireless sensor network nodes have limited energy, how to employ limited energy efficiently to realize effective data transmission has become a hot topic. Considering the characteristics of orchard planting in rows and shade caused by sparse random features, to improve energy efficiency of the orchard wireless sensor network and prolong network lifetime, we propose an improved chain-based clustering hierarchical routing (ICCHR) algorithm based on LEACH algorithm. The ICCHR algorithm investigates the formation of clusters, cluster head election, chain formation as well as the data transmission process, and further simulated with E-LEACH, PEGASIS-E, LEACH-1R PEGASIS and P-LEACH algorithms through MATLAB. The simulation results show that for BS at $(50,175)$, from the point of view of all sensor nodes death metric, the network lifetime for ICCHR algorithm prolongs about 3.29, 8.78, 35.53, and 43.11\% compared with E-LEACH, PEGASISE, LEACH-1R PEGASIS and P-LEACH algorithms. The average energy consumption per round of the ICCHR algorithm is lower than E-LEACH, PEGASIS-E, LEACH-1R PEGASIS and P-LEACH algorithms about 4.73, 9.04, 35.60, and 43.31\%. This research can provide theoretical references for the orchard complex environment wireless networking.
\end{abstract}

Keywords Orchard · Wireless sensor network · ICCHR algorithm · Network lifetime · Energy consumption load balancing

\section{Introduction}

Wireless sensor network (WSN), as a rapidly developing technology, has been widely applied in health monitoring, environmental monitoring, precision agriculture, and so on $[1,2]$. With the improvement of people's living standards and living quality, the demand of economic crops becomes larger and larger [3]. Apple, as one of the most important economic crops in China, the precise management of apple orchard has an important role in promoting the

Huaji Zhu

nercitasoft@126.com

1 National Engineering Research Center for Information Technology in Agriculture, Beijing 100097, People's Republic of China

2 Beijing Research Center for Information Technology in Agriculture, Beijing Academy of Agriculture and Forestry Sciences, Beijing 100097, People's Republic of China

3 Key Laboratory of Agri-informatics, Ministry of Agriculture, Beijing 100097, People's Republic of China

4 Key Laboratory of Agricultural Internet of Things, Ministry of Agriculture and Rural Affairs, Yangling, China economic growth of China [4]. Given the characteristics of WSN, orchard environment monitoring based on WSN has attracted widespread attention.

Generally, the sensor nodes in a WSN are battery powered and difficult to replace after deployment, so the sensor nodes have very limited energy. If the sensor nodes in the network are unable to work due to energy exhaustion, the network topology will be changed [5] and the routing will be re-established [6]. Therefore, how to effectively utilize the limited battery energy of network without affecting the function becomes the key issue to be considered in network design [7-14]. At present, a lot of domestic and international researches have been done on WSN routing protocols [15-28], and found that a better route can prolong the network lifetime and improve the network stability. Hierarchical routing protocols can provide good scalability for numerous sensor nodes, and can realize the data aggregation through cluster head $(\mathrm{CH})$ in each cluster [29]. For example, an E-LEACH protocol was proposed in which the remnant power of the sensor nodes and optimal cluster size are taken to balance network loads and change the round time [30]. The PEGASIS-E protocol was proposed in which 
the average distance among the sensor nodes is utilized as the criteria for chaining [31].

In apple orchard, the fruit trees are planted in rows that are spaced 3-4 m apart. Different from other monitoring regions, the sensor nodes are deployed based on the locations of fruit trees. Considering the distribution characteristics of deployed sensor nodes, it is essential to investigate the routing protocol in orchard environment. It is noted that very little research has focused on investigating routing protocol in orchard environment. In this paper, based on LEACH algorithm, an improved chain-based clustering hierarchical routing (ICCHR) is proposed, which adapts to the monitoring environment of orchard line planting. In this protocol, the $\mathrm{CH}$ nodes do not directly transmit signals to BS, but transmit them to the outside one by one by using the chain method, which overcomes the problem that the internal nodes die too fast. At the same time, a new $\mathrm{CH}$ competition parameter is adopted in the selection of $\mathrm{CH}$ to reduce the energy consumption of the network and improve the stability. The findings in this research can provide references for WSN networking in complex orchard environment.

The paper can be organized as follows: Sect. 2 presents the detailed study of routing based clustering protocol. Section 3 presents advantages and inefficiency of the typical hierarchical routing algorithms. The proposed ICCHR algorithm is described in Sect. 4. The performance analysis and results discussion are drawn in Sect. 5. Section 6 concludes the paper.

\section{Related Works}

Recently, there have been many researches focused on investigating the energy-efficient routing protocols. For example, in [32], a new protocol called ECHERP which can pursue energy conservation through balanced clustering has been proposed. It is found that the proposed protocol is efficient in energy consumption. In [33], the EDAL protocol which can reduce computational overhead has been proposed. It is found the proposed protocol can achieve considerable reduction in total traffic cost for collecting sensor readings under loose delay bounds. In [34], the M-ATTEMPT protocol has been proposed. It is found that the proposed protocol has less energy consumption and more reliable as compared to Multi-hop communication. In [35], the routing protocol was developed with an efficient particle encoding scheme and multi-objective fitness function. It is found that the proposed protocol can perform better in terms of network lifetime, energy consumption and delivery of data packets to the base station. In [36], a new energy-efficient routing protocol using message success rate has been proposed. It is found that the protocol can outperform the existing schemes in terms of communication reliability and energy efficiency. In [37], the DVRP protocol in which the forwarding of data packets is based on the flooding zone angle by the sender nodes toward the surface sink has been proposed. It is found that the proposed protocol can perform better in terms of endto-end delays, energy consumption and data delivery ratios. In [38], the DFCR protocol which adopting a distributed run time recovery of the sensor nodes due to sudden failure of the $\mathrm{CHs}$ has been proposed. It is found that the proposed protocol is energy efficient and fault tolerant. In [39], a cluster-based routing protocol for wireless sensor networks with nonuniform node distribution has been proposed. It is found that the proposed protocol can balance the energy consumption among nodes and increase the network lifetime significantly. In [40], the BEENISH protocol which assuming WSN containing four energy levels of nodes has been proposed. It is found that the proposed protocol can achieve longer stability, lifetime and more effective messages. In [41], the EDDEEC protocol which electing $\mathrm{CH}$ based on changing dynamically probability has been proposed. It is found that the proposed protocol can present longer lifetime, stability period and more effective messages to BS than DEEC. In [42], the ESRPSDC routing protocol which adopting error recovery to avoid end-to-end error recovery has been proposed. It is found that the proposed protocol can significantly improve the energy efficiency and packet reception rate. In [43], an EELBC algorithm that addressing energy efficiency as well as load balancing has been proposed. It is found that the proposed algorithm can perform better in terms of load balancing, energy efficiency, and execution time. In [44], the EHGUC-OAPR algorithm which combining the energy harvesting genetic-based unequal clustering algorithm and optimal adaptive performance routing algorithm has been proposed. It is found that the proposed algorithm has a great improvement in network energy balance and data delivery ratio.

\section{The Typical Hierarchical Routing Algorithms and Existing Problems}

Researches on hierarchical routing algorithms of WSN has made some progress, among which LEACH and PEGASIS algorithms are the most typical.

\subsection{E-LEACH Protocol Algorithm}

The E-LEACH algorithm adopts the same round concept with the original LEACH [30], and each round can be divided into clustering phase and stable transmission phase. In the clustering stage, each node that has not served as the $\mathrm{CH}$ produces a random number between 0 and 1 . If the generated random number is less than the given threshold value $T(n)$, the node 
is elected as the $\mathrm{CH}$. The threshold $T(n)$ can be calculated as follows:

$T(n)=\left\{\begin{array}{ll}\frac{P}{1-P \times[r \bmod (1 / P)]} \times \frac{E_{\text {residual }}}{E_{0}} & n \in G \\ 0 & n \notin G\end{array}\right.$,

where $P$ is the expected percentage to become the $\mathrm{CH}, r$ is the current number of rounds, $E_{\text {residual }}$ is the residual energy of nodes at the $r$ round, $E_{0}$ is the initial energy of nodes, and $G$ is the node set that has not become the $\mathrm{CH}$ in the last $1 / P$ round.

In the stable transmission phase, the member nodes transmit data to the $\mathrm{CH}$ according to TDMA time slot allocated. The $\mathrm{CH}$ integrates the received data and transfers it to the sink node.

\subsection{PEGASIS-E Protocol Algorithm}

PEGASIS-E is a improved chain based routing algorithm which operates in round [31], and each round can be divided into chain construction phase, leader selection phase, and data transmission phase. In the chain construction phase, the node farthest from BS join the chain first till all the nodes join the chain. In the leader selection phase, the leader in each round of communication is at the random location on the chain which ensuring robustness of network towards failures [31]. In the data transmission phase, each node delivers its own sensed data to its neighbor, and the neighbor nodes further fuse the received data with their own data and forwards further towards the leader [31].

\section{The ICCHR Algorithm}

\subsection{Network Model and Assumptions}

In this paper, there are $\mathrm{N}$ sensor nodes randomly arranged in a $\mathrm{M} \times \mathrm{M}$ square area. Moreover, some assumptions are made as follows: the sensor nodes and BS are at the static; the energy of sink node is unlimited and that of common nodes are energyconstrained; all sensor nodes location is known; Links are symmetric.

\subsection{Energy Consumption Model}

Herein, we adopt the First-order Radio Model [30] which has been employed in many researches $[29,31]$ as the energy model. For $k$-bit messages transmission, the energy consumed can be calculated as follows:

$E_{T x}(k, d)=\left\{\begin{array}{ll}k E_{\text {elec }}+k E_{f s} d^{2} & d<d_{0} \\ k E_{\text {elec }}+k E_{\text {amp }} d^{4} & d \geq d_{0}\end{array}\right.$,

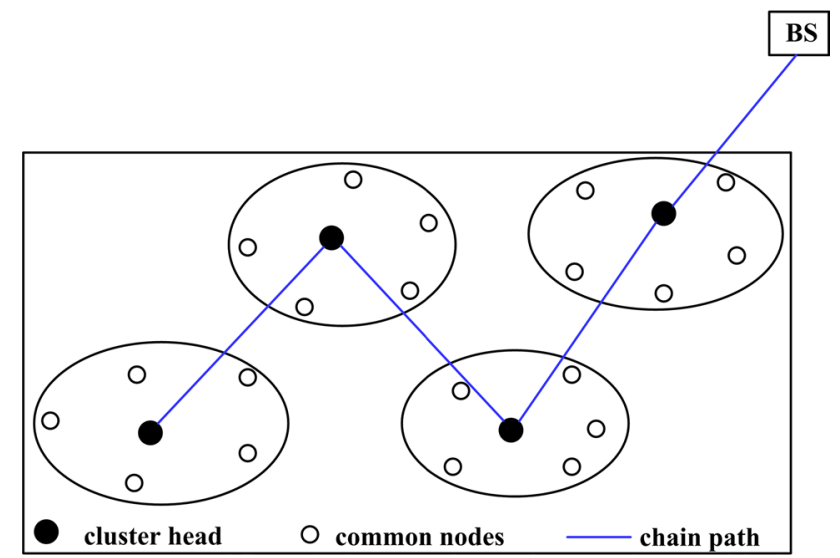

Fig. 1 The architecture of ICCHR algorithm

where $k$ is the number of messages, $d$ is the distance from transmitter, and $E_{\text {elec }}$ is the amount of energy consumed in electronics. Moreover, $E_{a m p}$ and $E_{f s}$ are the energy consumed in amplifiers. The energy expended in receiving $k$-bit messages can be calculated as follows:

$E_{R x}(k)=k E_{\text {elec }}$.

The energy consumed for fusion of $l$ length of $k$ bits data packet can be calculated as follows:

$E_{R x}=k l E_{D F}$,

where $E_{D F}$ is the energy required to fuse the data per bit.

\subsection{The Description of ICCHR Algorithm}

The implementation process of ICCHR algorithm is periodic, and each round can be divided into two stages, i.e., the formation of cluster and stable data communication. The network architecture is presented in Fig. 1.

\subsubsection{CH Election}

In order to resolve the deficiency of LEACH algorithm, the ICCHR algorithm adopts a threshold setting method which is applicable for orchard long direct deployment environment:

$T(n)=\left\{\begin{array}{ll}\frac{P}{1-P \times[r \bmod (1 / P)]} \times\left(\frac{E_{\text {initial }}}{E(i)_{r}}\right) \times\left(1-\frac{D_{2}(i)}{D(i)}\right) \times \frac{D_{1}(i)}{D(i)} & n \in G \\ 0 & n \notin G\end{array}\right.$,

where $E(i)_{r}$ is the residual energy of sensor node, $E_{\text {initial }}$ is the initial energy of sensor node, $D_{l}(i)$ is the sum of the distances between sensor node $i$ and other sensor nodes, and $D_{2}(i)$ is the distance between sensor node $i$ and sink node. Moreover, $D(i)$ can be calculated as follows:

$D(i)=\omega \times D_{1}(i)+(1-\omega) \times D_{2}(i)$, 
where $\omega \in[0,1]$. If the generated random number of sensor node $\mathrm{i}$ is smaller than the calculated threshold, the sensor node $\mathrm{i}$ is elected as $\mathrm{CH}$.

\subsubsection{The Formation of Cluster}

To balance the energy consumption of $\mathrm{CH}$, we adopt the nonuniform clustering algorithm.

$\bar{D}=\frac{d\left(N_{k}, C_{i}\right)}{d_{\max }\left(N, C_{i}\right)}+\left(1-\frac{d\left(C_{i}, B S\right)}{d_{\max }(C, B S)}\right)$,

where $d\left(N_{k}, C_{i}\right)$ represents the distance from cluster members to $\mathrm{CH}, d_{\max }\left(N, C_{i}\right)$ indicates the maximum distance from cluster members to $\mathrm{CH}, d\left(C_{i}, B S\right)$ represents the distance from $\mathrm{CH}$ to $\mathrm{BS}$, and $d_{\max }(C, B S)$ indicates the maximum distance from $\mathrm{CH}$ to $\mathrm{BS}$. After receiving the broadcast sent by the $\mathrm{CH}$, the node selects the $\mathrm{CH}$ owning the smallest $\bar{D}$ to join. When the node selects the cluster it belongs to, the $\mathrm{CH}$ receives the ID of the cluster members as well as the distance between the cluster members and $\mathrm{CH}$, and then $\mathrm{CH}$ assigns TDMA to the cluster members. The cluster members continuously collect monitoring data, and then send the data to the $\mathrm{CH}$ for aggregation.

\subsubsection{Chain Clustering Routing Mechanism}

The elected CHs employ greedy algorithm to be a chain, and the $\mathrm{CH}$ farthest from the sink node can be labeled as the current access node. Then, the $\mathrm{CH}$ farthest from the sink node which has not been labeled can be set as the neighbor node, and further be labeled as the current access node. And so on, until all $\mathrm{CHs}$ are labeled, indicating that all $\mathrm{CHs}$ are on the chain. Considering that the forwarding tasks undertaken by the chain leader are the most numerous and the energy consumption is the fastest, the $\mathrm{CH}$ closest to the BS is selected as the chain header to directly communicate with the BS. Figures 2 and 3 are the pseudocode description and the flow chart of the ICCHR algorithm. As can be seen, first of all, all sensor nodes send their location and energy information to the BS. Then, the nodes with the shortest distance from other nodes, the farthest distance from the BS and the maximum residual energy in the cluster are elected as CHs. Next, the BS notifies the $\mathrm{CH}$ information to the cluster members, and the cluster members then send the confirmation information to the BS. All the elected $\mathrm{CH}$ s form a chain, and the $\mathrm{CH}$ s with the shortest distance from the BS are elected as the chain leader which can directly communicate with the BS.

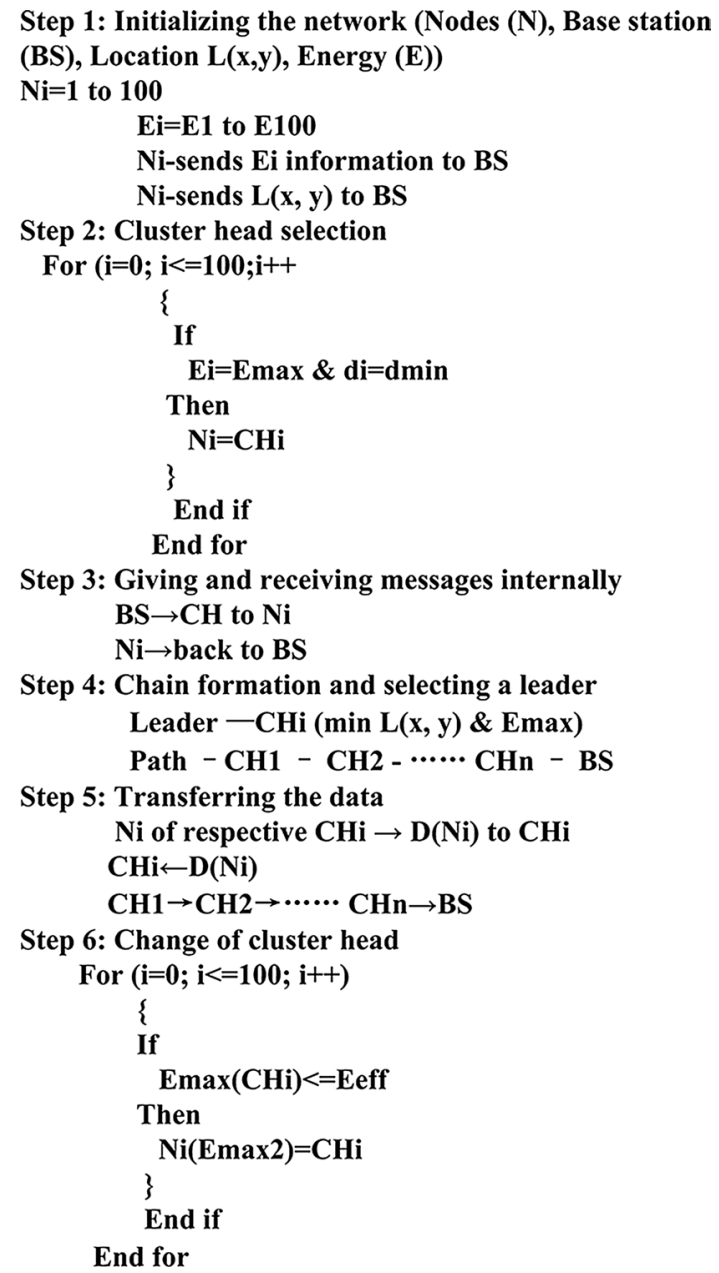

Fig. 2 The pseudocode description of ICCHR algorithm

\subsection{Stable Transmission Phase}

\subsubsection{Communications Within the Clusters}

The cluster members only send messages to the $\mathrm{CH}$, and there is no forwarding process of the cluster members, which reducing the energy consumption of controlling information transmission. The $\mathrm{CH}$ receives the information sent by the cluster members, and the fusion of information will be shown.

\subsubsection{Inter-cluster Communication}

All information between clusters shall be aggregated along the chain to the chain leader after fusion, and then forwarded to the BS. For the stable communication stage, in the last round, all cluster members send their residual energy to the $\mathrm{CH}$ which is sent to the BS through the chain, and then the process of re-electing the $\mathrm{CH}$ in the next round will begin. The data transmission process as presented in Fig. 4 can be 


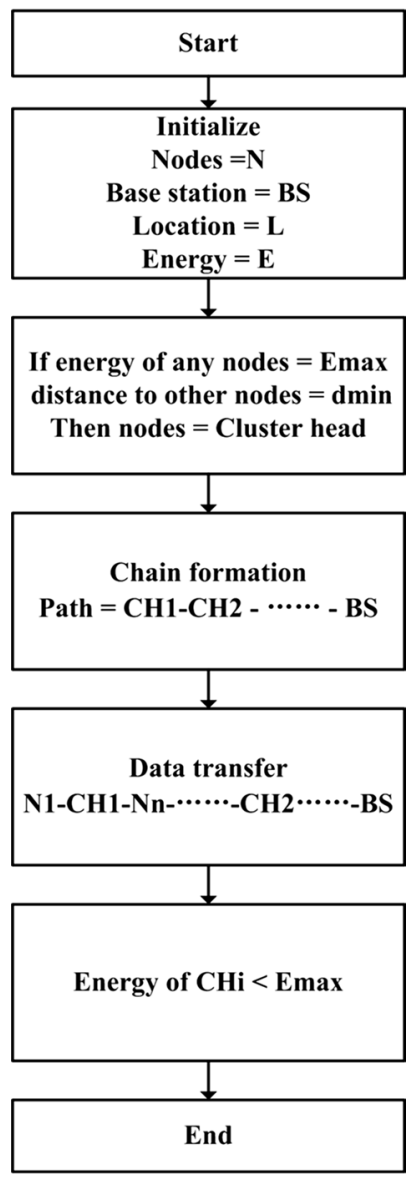

Fig. 3 The flow chart of ICCHR algorithm

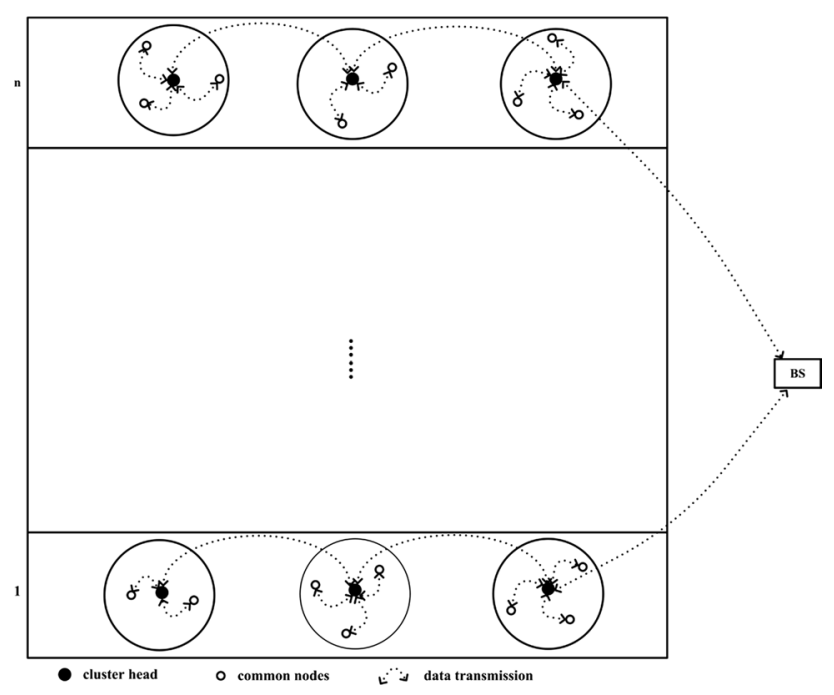

Fig. 4 The data transmission process of ICCHR algorithm
Table 1 The simulation parameters

\begin{tabular}{ll}
\hline Parameters & Value \\
\hline Network coverage area/ $/ \mathrm{m} \times \mathrm{m})$ & $100 \times 100$ \\
Number of nodes & 100 \\
Coordinates of BS/(m, m) & $(0,0),(50$, \\
& $50),(50$, \\
Initial energy of nodes $/(\mathrm{J})$ & $175)$ \\
$E_{\text {elec }} /(\mathrm{nJ} / \mathrm{bit})$ & 0.5 \\
$E_{\mathrm{fs}} /\left(\mathrm{pJ} / \mathrm{bit} / \mathrm{m}^{2}\right)$ & 50 \\
$E_{\mathrm{amp}} /\left(\mathrm{pJ} / \mathrm{bit} / \mathrm{m}^{4}\right)$ & 100 \\
$E_{\mathrm{DA}} /(\mathrm{nJ} / \mathrm{bit})$ & 0.0013 \\
$\mathrm{Packet}$ length/bit & 5 \\
$P$ & 2000 \\
$\omega$ & 0.05 \\
$r$ & 0.1 \\
\hline
\end{tabular}

described as follows: cluster members send data to the corresponding $\mathrm{CH}$, and the $\mathrm{CH}$ transfers data along the optimal path depicted in Fig. 1 and converges to the chain leader which can directly communicate with the BS.

\section{Simulation and Results}

\subsection{Simulation Setup}

The simulations are performed in an area of $100 \mathrm{~m} \times 100 \mathrm{~m}$ with 100 sensor nodes randomly distributed. The location of BS is set to $(0,0),(50,50)$, and $(50,175)$, respectively. The initial energy of each sensor node is equal to $0.5 \mathrm{~J}$, and each node can transmit 2000 bits messages. For each node sends or receives data, the energy required by the transmitting circuit is $E_{\text {elec }}=50 \mathrm{~nJ} / \mathrm{bit}$, the energy consumed by the power amplifier is $\mathrm{E}_{\mathrm{fs}}=100 \mathrm{pJ} /\left(\mathrm{bit}^{2}\right)$, the energy consumed by the $\mathrm{CH}$ data fusion is $\mathrm{E}_{\mathrm{DA}}=5 \mathrm{npJ} / \mathrm{bit}$, the amplification factor of the signal amplifier is $\mathrm{E}_{\mathrm{amp}}=0.0013 \mathrm{pJ} / \mathrm{bit} / \mathrm{m}^{4}$, the signal transmission distance $\mathrm{d}_{0}=87 \mathrm{~m}$, and the sampling period is $10 \mathrm{~s}$. The detailed simulation parameters are listed in Table 1.

\subsection{Performance Analysis}

To evaluate the performance of the proposed ICCHR algorithm, the simulations are performed by MATLAB and compared with E-LEACH, PEGASIS-E, LEACH-1R PEGASIS [45], and P-LEACH [46] algorithms. The performance metrics include network lifetime, throughput, even distribution of energy usage, and time complexity. 


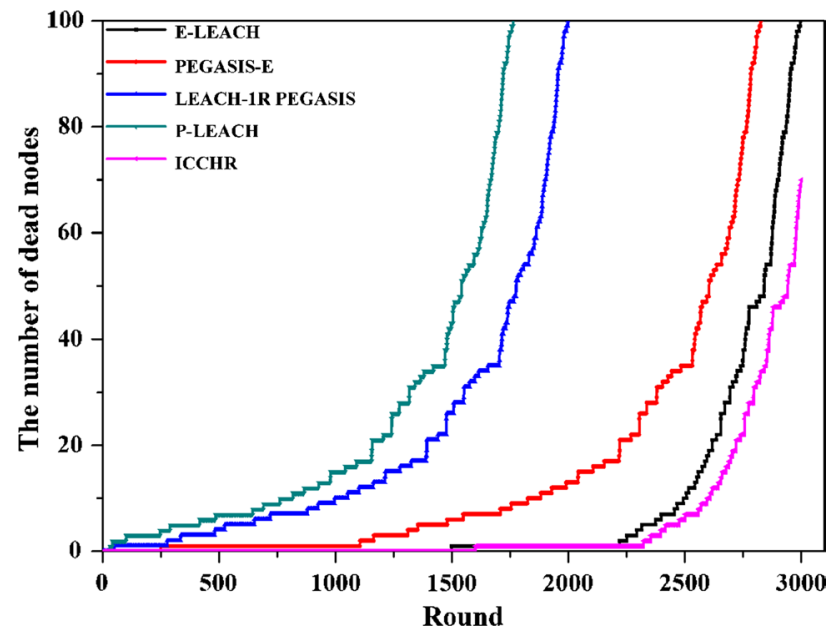

Fig. 5 The number of dead nodes varying with rounds for BS at (50, 175)

\subsubsection{Network Lifetime}

Figure 5 shows the network lifetime diagram for E-LEACH algorithm, PEGASIS-E algorithm, LEACH-1R PEGASIS algorithm, P-LEACH algorithm, and the proposed ICCHR algorithm. As can be seen, the first node death occur in the 1497 round, and all the nodes death is at about 2997 round in E-LEACH algorithm. The first node death in PEGASIS-E algorithm is at 247 round, and all the nodes death is at about 2627 round. For the LEACH-1R PEGASIS algorithm, the first node death is at about 47 round, and all the nodes death occur at 1998 round. For the P-LEACH algorithm, the first node death is at about 38 round, and all the nodes death occur at 1763 round. While for the proposed ICCHR algorithm, the first node death appears in 1600 round, and all the nodes death is at about 3099 round. Obviously, the proposed ICCHR algorithm can greatly delay the round of node death. Moreover, the changing curve of the proposed ICCHR algorithm is relatively smooth, indicating that ICCHR algorithm is more stable than E-LEACH, PEGASIS-E, LEACH-1R PEGASIS, and P-LEACH algorithms.

To describe the effect of the BS location on network lifetime, the comparisons of five algorithms using first node death (FND), half of nodes death (HND), and last node death (LND) three metrics with BS locations at $(0,0)$, $(50,50)$, and $(50,175)$ are illustrated in Fig. 6. As can be seen, for BS at $(0,0)$, from the point of view of FND, the proposed ICCHR algorithm extends the network lifetime approximately by $6.30,84.49,97.00$, and $97.46 \%$ compared with E-LEACH, PEGASIS-E, LEACH-1R PEGASIS, and P-LEACH algorithms, respectively. From perspective of HND, the proposed ICCHR algorithm extends the network lifetime approximately by $4.05,11.53,39.70$, and $47.69 \%$ compared with E-LEACH, PEGASIS-E, LEACH-1R
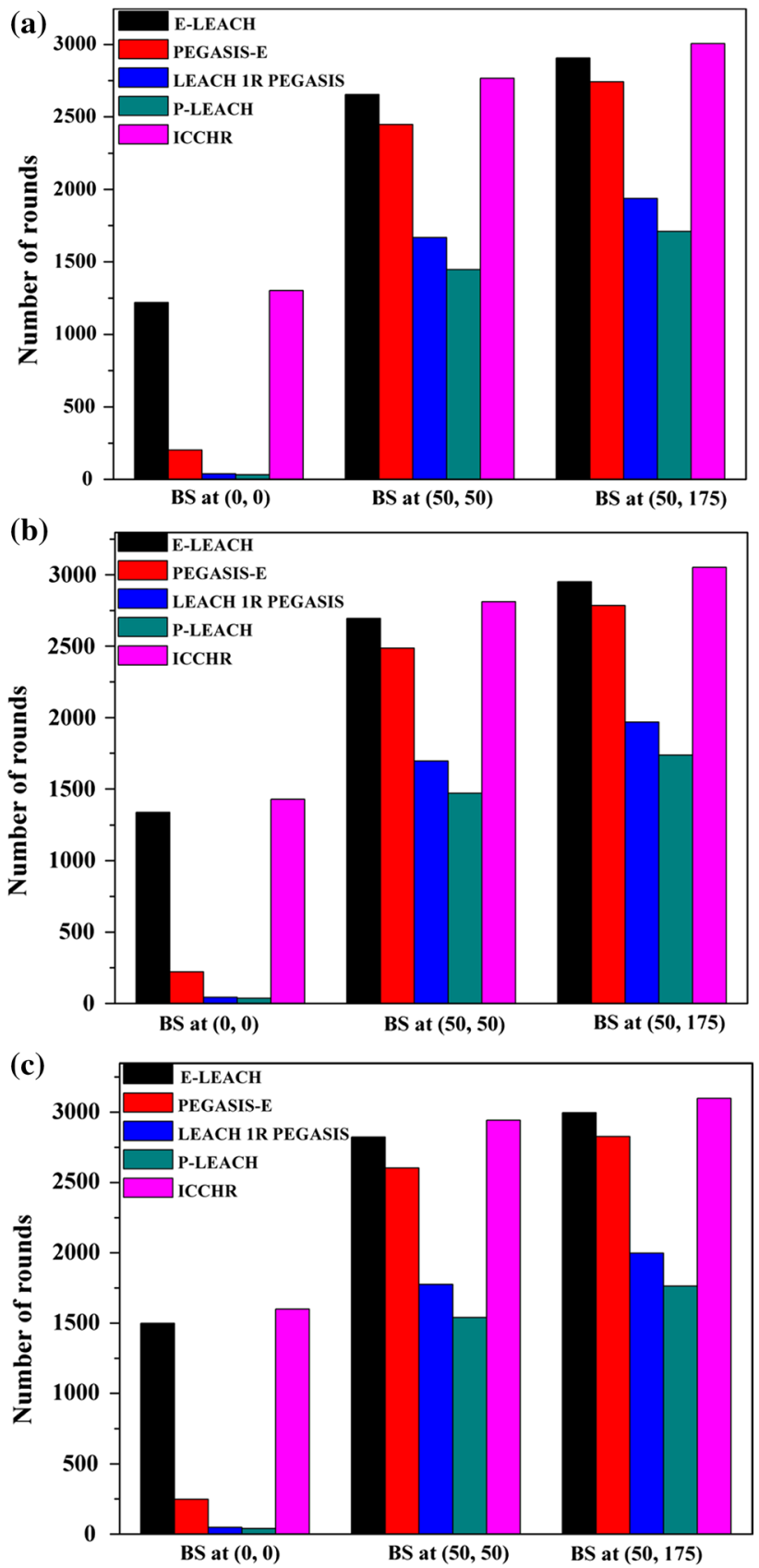

Fig. 6 The comparisons of five algorithms using FND, HND, and AND three metrics for BS locations at $(0,0),(50,50)$, and $(50,175)$

PEGASIS, and P-LEACH algorithms, respectively. From perspective of AND, the proposed ICCHR algorithm extends the network lifetime approximately by 3.29, 8.78, 35.53, and $43.11 \%$ in comparison with E-LEACH, PEGASIS-E, LEACH-1R PEGASIS, and P-LEACH algorithms, respectively. For BS at $(50,50)$, the proposed ICCHR algorithm extends the network lifetime approximately by $6.30,84.52$, 96.99 and $97.48 \%$ compared with E-LEACH, PEGASIS-E, LEACH-1R PEGASIS and P-LEACH algorithms in terms 


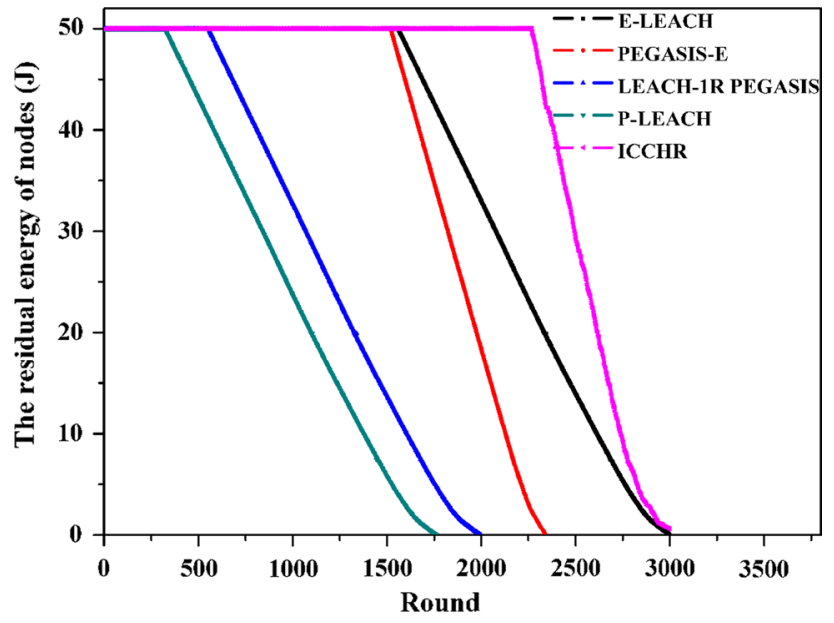

Fig. 7 The residual energy of sensor nodes varying with rounds for BS at $(50,175)$

of FND. For HND, the proposed ICCHR algorithm exhibits the longer network lifetime approximately by $4.13,11.53$, 39.70 and $47.71 \%$ compared with E-LEACH, PEGASISE, LEACH-1R PEGASIS and P-LEACH algorithms. From the point of view of AND, the proposed ICCHR algorithm extends the network lifetime approximately by $3.31,9.43$, 35.54 , and $43.11 \%$ compared with E-LEACH, PEGASISE, LEACH-1R PEGASIS, and P-LEACH algorithms, respectively. For BS at $(50,175)$, the proposed ICCHR algorithm extends the network lifetime in terms of FND approximately by $6.31,84.50,97.00$, and $97.50 \%$ compared with E-LEACH, PEGASIS-E, LEACH-1R PEGASIS and P-LEACH algorithms. From perspective of HND, the proposed ICCHR algorithm extends the network lifetime approximately by $4.08,11.52,39.69$ and $47.67 \%$ in contrast with E-LEACH, PEGASIS-E, LEACH-1R PEGASIS and P-LEACH algorithms. From perspective of AND, the proposed ICCHR algorithm extends the network lifetime approximately by $3.29,8.78,35.53$, and $43.11 \%$ compared with E-LEACH, PEGASIS-E, LEACH-1R PEGASIS and P-LEACH algorithms. Therefore, the proposed ICCHR algorithm is significantly superior to the other four algorithms in terms of the number of communication rounds corresponding to either first node death or the last node death and the communication interval between them. The results show that the proposed ICCHR algorithm has higher energy utilization, more balanced load and longer life cycle.

\subsubsection{Even Distribution of Energy Usage}

The comparisons of the residual energy varying with the rounds for BS at $(50,175)$ are presented in Fig. 7. As can be seen, the residual energy in the proposed ICCHR algorithm is higher than that in E-LEACH, PEGASIS-E, LEACH-1R
Table 2 The average energy consumed per round for BS at $(0,0),(50$, $50)$ and $(50,175)$

\begin{tabular}{llllll}
\hline \begin{tabular}{l} 
BS $\begin{array}{l}\text { coordi- } \\
\text { nates/ } \\
(\mathrm{m}, \mathrm{m})\end{array}$ \\
\cline { 2 - 6 }
\end{tabular} & $\begin{array}{l}\text { E-LEACH } \\
\text { The average energy consumed per round/(J) }\end{array}$ & $\begin{array}{l}\text { PEGASIS- } \\
\text { E }\end{array}$ & $\begin{array}{l}\text { LEACH- } \\
\text { 1R } \\
\text { PEGASIS }\end{array}$ & P-LEACH & ICCHR \\
\hline$(0,0)$ & 0.0172 & 0.0182 & 0.0258 & 0.0292 & 0.0166 \\
$(50,50)$ & 0.0169 & 0.0181 & 0.0254 & 0.0288 & 0.0164 \\
$(50$, & 0.0169 & 0.0177 & 0.0250 & 0.0284 & 0.0161 \\
$175)$ & & & & & \\
\hline
\end{tabular}

PEGASIS and P-LEACH algorithms at the same round. Especially at round 2997, the residual energy in E-LEACH, PEGASIS-E, LEACH-1R PEGASIS and P-LEACH algorithms is close to zero, but that in the proposed ICCHR algorithm is approximately $0.6 \mathrm{~J}$. In the initial stage of simulation, there is no obvious differences in energy consumption among five algorithms. As the number of rounds increases, the ICCHR algorithm exhibits significant advantages in extending the network lifetime, which is consistent with the analysis of alive nodes. This phenomenon can be attributed to the ICCHR algorithm employing chain transmission among clusters, further avoiding the shortages of communication between each CH and BS in E-LEACH algorithm and the long distance transmission in PEGASIS-E algorithm. The average energy consumed per round for BS at $(0,0)$, $(50,50)$ and $(50,175)$ is listed in Table 2 . As can be seen, for BS at $(0,0)$, the proposed ICCHR algorithm consumes less energy compared with E-LEACH, PEGASIS-E, LEACH-1R PEGASIS and P-LEACH algorithms approximately by 3.49 , $8.79,35.66$, and $43.15 \%$, respectively. For BS at $(50,50)$, the proposed ICCHR algorithm consumes less energy compared with E-LEACH, PEGASIS-E, LEACH-1R PEGASIS and P-LEACH algorithms by 2.96, 9.39, 35.43 and $43.06 \%$, respectively. While for BS at $(50,175)$, the proposed ICCHR algorithm consumes less energy compared with E-LEACH, PEGASIS-E, LEACH-1R PEGASIS and P-LEACH algorithms approximately by $4.73,9.04,35.60$, and $43.31 \%$, respectively. Obviously, the proposed ICCHR algorithm consumes less energy per round compared with the other four algorithms, indicating that the ICCHR algorithm is more sustainable and energy saving.

\subsubsection{Throughput}

Figure 8 depicts the number of data messages received by BS varying with rounds. Compared with the E-LEACH, PEGASIS-E, LEACH-1R PEGASIS and P-LEACH algorithms, the data messages received by $\mathrm{BS}$ for the proposed ICCHR algorithm is greater. The reason can be ascribed to 


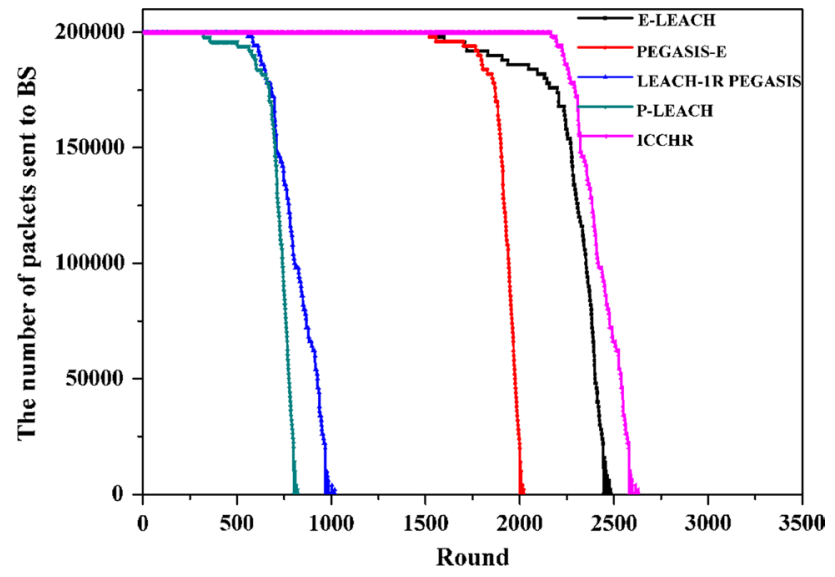

Fig. 8 The number of packets sent to BS varying with rounds for BS at $(50,175)$

Table 3 The time complexity for E-LEACH, PEGASIS-E, LEACH-1R PEGASIS and P-LEACH algorithms

\begin{tabular}{ll}
\hline Time complexity & Value \\
\hline E-LEACH & $\mathrm{O}(\mathrm{L})$ \\
PEGASIS-E & $\mathrm{O}(\mathrm{L})^{\mathrm{n}}$ \\
LEACH-1R PEGASIS & $2 \mathrm{nO}(\mathrm{L})$ \\
P-LEACH & $\mathrm{nO}(\mathrm{L})$ \\
ICCHR & $\mathrm{O}(\mathrm{L})$ \\
\hline
\end{tabular}

the extending network lifetime and more balancing energy consumption.

\subsubsection{Time Complexity}

Time complexity analysis of five algorithms is illustrated in Table 3. As can be seen, $\mathrm{O}(\mathrm{L})$ explains the time complexity of solving a linear optimization problem. The simulation results illustrate that the proposed ICCHR algorithm has lower time complexity compared with PEGASIS-E, LEACH-1R PEGASIS and P-LEACH algorithms.

\section{Conclusion}

The WSN architecture suitable for orchard information acquisition is constructed, and the hierarchical routing is adopted to achieve energy consumption load balancing and prolonging network lifetime. Based on the analysis of ICCHR algorithm, the clustering formation, the election of $\mathrm{CH}$, chain formation and the data transmission process are described in detail, and the simulation results are given. According to the analysis of simulation results, for BS at (50, 175), from the prospective of AND, the proposed ICCHR algorithm extends the network lifetime approximately by $3.29,8.78,35.53$, and $43.11 \%$ compared with E-LEACH,
PEGASIS-E, LEACH-1R PEGASIS and P-LEACH algorithms, respectively. Moreover, the proposed ICCHR algorithm consumes less energy compared with E-LEACH, PEGASIS-E, LEACH-1R PEGASIS and P-LEACH algorithms approximately by $4.73,9.04,35.60$ and $43.31 \%$, making the energy consumption load more balancing.

Acknowledgements This work was supported by Natural Science Foundation of China (61571051), Beijing Natural Science Foundation (4172024), and Opening Foundation of Key Laboratory of Agricultural Internet of Things, Ministry of Agriculture and Rural Affairs (2018AIOT-06).

Open Access This article is distributed under the terms of the Creative Commons Attribution 4.0 International License (http://creativeco mmons.org/licenses/by/4.0/), which permits unrestricted use, distribution, and reproduction in any medium, provided you give appropriate credit to the original author(s) and the source, provide a link to the Creative Commons license, and indicate if changes were made.

\section{References}

1. Pellegrini A, Brizzi A, Zhang L et al (2013) Antennas and propagation for body-centric wireless communications at millimeter-wave frequencies: a review. IEEE Antennas Propag Mag 55(4):262-287

2. Kim YD, Yang YM, Kang WS et al (2014) On the design of beacon based wireless sensor network for agricultural emergency monitoring systems. Comput Stand Interfaces 36(2):288-299

3. Boserup E (2017) The conditions of agricultural growth: the economics of agrarian change under population pressure. Routledge, Abingdon

4. Rao Y, Jiang ZH, Lazarovitch N (2016) Investigating signal propagation and strength distribution characteristics of wireless sensor networks in date palm orchards. Comput Electr Agric 124:107-120

5. Zhang DG, Zhu YN, Zhao CP et al (2012) A new constructing approach for a weighted topology of wireless sensor networks based on local-world theory for the internet of things (IOT). Comput Math Appl 64(5):1044-1055

6. Nguyen HT, Ramstad TA, Balasingham I (2012) An adaptive transmission scheme for wireless sensor networks over slow fading channels. In: Computing technology and information management (ICCM), 2012 8th International Conference on IEEE, vol 2, pp 617-621

7. Ndzia DL, Harun A, Ramli FM, Kamarudin ML, Zakaria A, Shakaff AYM et al (2014) Wireless sensor network coverage measurement and planning in mixed crop farming. Comput Electr Agric 105:83-94

8. Zhang D, Wang $X$, Song $X$ (2015) New medical image fusion approach with coding based on SCD in wireless sensor network. J Electr Eng Technol 10(6):2384-2392

9. Zhang D, Wang X, Song X et al (2014) A novel approach to mapped correlation of ID for RFID anti-collision. IEEE Trans Serv Comput 7(4):741-748

10. Zhang DG, Zhang XD (2012) Design and implementation of embedded un-interruptible power supply system (EUPSS) for web-based mobile application. Enterp Inform Syst 6(4):473-489

11. Zhang D (2012) A new approach and system for attentive mobile learning based on seamless migration. Appl Intell 36(1):75-89 
12. Zhang D, Zheng K, Zhao DX et al (2016) Novel quick start (QS) method for optimization of TCP. Wirel Netw 22(1):211-222

13. Zhang D, Zhang T, Zhang J et al (2018) A kind of effective data aggregating method based on compressive sensing for wireless sensor network. EURASIP J Wirel Commun Netw 2018(1):159

14. Zhang D, Zhou S, Tang Y (2018) A low duty cycle efficient MAC protocol based on self-adaption and predictive strategy. Mob Netw Appl 23(4):828-839

15. Zhang D, Zhang T, Liu X (2018) Novel self-adaptive routing service algorithm for application in VANET. Appl Intell 49(5):1866-1879

16. Zhang D, Li G, Zheng K et al (2014) An energy-balanced routing method based on forward-aware factor for wireless sensor networks. IEEE Trans Indus Inform 10(1):766-773

17. Zhang DG, Zheng $\mathrm{K}$, Zhang $\mathrm{T}$ et al (2015) A novel multicast routing method with minimum transmission for WSN of cloud computing service. Soft Comput 19(7):1817-1827

18. Zhang D, Zhang T, Dong Y et al (2018) Novel optimized link state routing protocol based on quantum genetic strategy for Mobile learning. J Netw Comput Appl 122:37-49

19. Zhang D, Ge H, Zhang T et al (2018) New multi-hop clustering algorithm for vehicular ad hoc networks. IEEE Trans Intell Transp Syst 99:1-14

20. Zhang D, Liu S, Zhang T et al (2017) Novel unequal clustering routing protocol considering energy balancing based on network partition \& distance for mobile education. J Netw Comput Appl 88:1-9

21. Zhang D, Niu H, Liu S (2017) Novel PEECR-based clustering routing approach. Soft Comput 21(24):7313-7323

22. Zhang D, Liu S, Liu X et al (2018) Novel dynamic source routing protocol (DSR) based on genetic algorithm-bacterial foraging optimization (GA-BFO). Int J Commun Syst 31(18):e3824

23. Zhang D, Tang Y, Cui Y et al (2018) Novel reliable routing method for engineering of internet of vehicles based on graph theory. Eng Comput 16(1):226-247

24. Liu S, Zhang D, Liu X et al (2019) Dynamic analysis for the average shortest path length of mobile ad hoc networks under random failure scenarios. IEEE Access. https://doi.org/10.1109/ACCES S.2019.2896699

25. Zhang D, Gao J, Liu X et al (2019) Novel approach of distributed $\&$ adaptive trust metrics for MANET. Wirel Netw. https://doi. org/10.1007/s11276-019-01955-2

26. Tunca C, Isik S, Donmez MY et al (2015) Ring routing: an energy-efficient routing protocol for wireless sensor networks with a mobile sink. IEEE Trans Mob Comput 14(9):1947-1960

27. Naranjo PGV, Shojafar M, Mostafaei H et al (2017) P-SEP: a prolong stable election routing algorithm for energy-limited heterogeneous fog-supported wireless sensor networks. J Supercomput 73(2):733-755

28. Zahedi ZM, Akbari R, Shokouhifar M et al (2016) Swarm intelligence based fuzzy routing protocol for clustered wireless sensor networks. Expert Syst Appl 55:313-328

29. Jan MA, Nanda P, He X et al (2013) Enhancing lifetime and quality of data in cluster-based hierarchical routing protocol for wireless sensor network. In: 2013 IEEE 10th international conference on high performance computing and communications \& 2013 IEEE international conference on embedded and ubiquitous computing, IEEE, pp 1400-1407

30. Xu J, Jin N, Lou X et al (2012) Improvement of LEACH protocol for WSN. In: 2012 9th international conference on fuzzy systems and knowledge discovery, IEEE, pp 2174-2177
31. Nehra V, Sharma AK (2013) PEGASIS-E: power efficient gathering in sensor information system extended. Glob J Comput Sci Technol 13(15):1-5

32. Nikolidakis S, Kandris D, Vergados D et al (2013) Energy efficient routing in wireless sensor networks through balanced clustering. Algorithms 6(1):29-42

33. Yao Y, Cao Q, Vasilakos AV (2015) EDAL: an energy-efficient, delay-aware, and lifetime-balancing data collection protocol for heterogeneous wireless sensor networks. IEEE/ACM Trans Netw (TON) 23(3):810-823

34. Javaid N, Abbas Z, Fareed MS et al (2013) M-ATTEMPT: a new energy-efficient routing protocol for wireless body area sensor networks. Procedia Comput Sci 19:224-231

35. Kuila P, Jana PK (2014) Energy efficient clustering and routing algorithms for wireless sensor networks: particle swarm optimization approach. Eng Appl Artif Intell 33:127-140

36. Yoon M, Kim YK, Chang J (2013) An energy-efficient routing protocol using message success rate in wireless sensor networks. JoC 4(1):15-22

37. Ali T, Jung LT, Faye I (2014) Diagonal and vertical routing protocol for underwater wireless sensor network. Procedia-Soc Behav Sci 129:372-379

38. Azharuddin M, Kuila P, Jana PK (2015) Energy efficient fault tolerant clustering and routing algorithms for wireless sensor networks. Comput Electr Eng 41:177-190

39. Yu J, Qi Y, Wang G et al (2012) A cluster-based routing protocol for wireless sensor networks with nonuniform node distribution. AEU-Int J Electr Commun 66(1):54-61

40. Qureshi TN, Javaid N, Khan AH et al (2013) BEENISH: balanced energy efficient network integrated super heterogeneous protocol for wireless sensor networks. Procedia Comput Sci 19:920-925

41. Javaid N, Qureshi TN, Khan AH et al (2013) EDDEEC: enhanced developed distributed energy-efficient clustering for heterogeneous wireless sensor networks. Procedia Comput Sci 19:914-919

42. Ganesh S, Amutha R (2013) Efficient and secure routing protocol for wireless sensor networks through SNR based dynamic clustering mechanisms. J Commun Netw 15(4):422-429

43. Kuila P, Jana PK (2012) Energy efficient load-balanced clustering algorithm for wireless sensor networks. Procedia Technol 6:771-777

44. Elbhiri B, Saadane R, Aboutajdine D (2010) Developed distributed energy-efficient clustering (DDEEC) for heterogeneous wireless sensor networks. In: I/V communications and mobile network (ISVC), 2010 5th international symposium on IEEE, pp 1-4

45. Boulhares I, Omari M (2016) Hybridizing PEGASIS with LEACH-1R protocols in wireless sensor networks. In: 2016 8th international conference on modelling, identification and control (ICMIC), IEEE, pp 1037-1042

46. Razaque A, Abdulgader M, Joshi C et al (2016) P-LEACH: energy efficient routing protocol for wireless sensor networks. In: 2016 IEEE long island systems, applications and technology conference (LISAT), IEEE, pp 1-5

Publisher's Note Springer Nature remains neutral with regard to jurisdictional claims in published maps and institutional affiliations. 


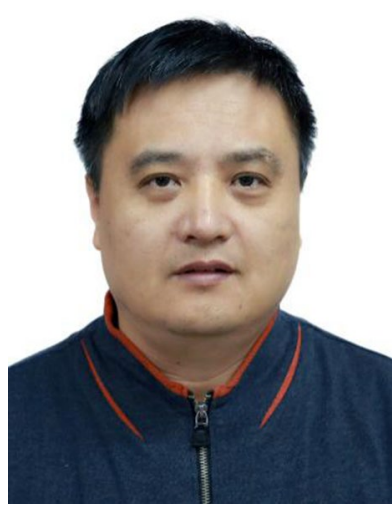

Huarui $\mathbf{W u}$ is a professor at National Engineering Research Center for Information Technology in Agriculture. He is interested in studying artificial intelligence.

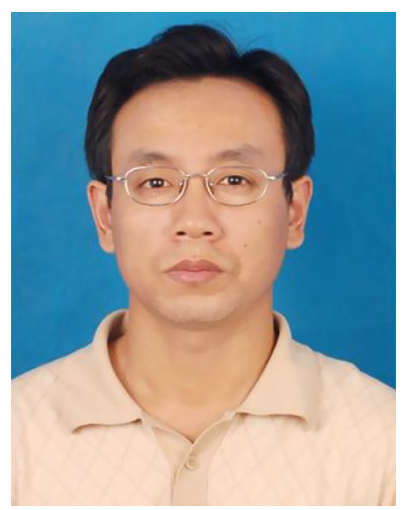

Huaji Zhu is a professor at National Engineering Research Center for Information Technology in Agriculture. He is interested in studying agricultural internet of things technology.

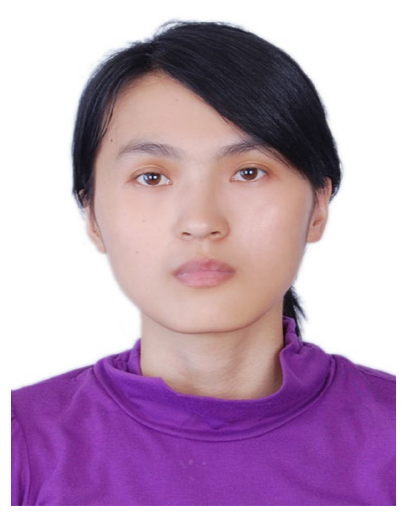

Lihong Zhang is an engineer at National Engineering Research Center for Information Technology in Agriculture. Her research interest is internet of things technology.

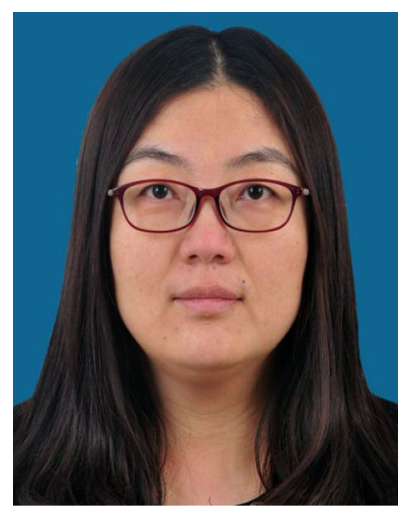

Yuling Song is an experimenter at the College of Mechanical and Electrical Engineering of Northwest A\& F University. Her research interest is non-linear dynamics analysis. 